\title{
Ground-state correlations in semiconductor double-quantum-wire systems
}

\author{
N. Mutluay and B. Tanatar \\ Department of Physics, Bilkent University, Bilkent, 06533 Ankara, Turkey
}

(Received 20 June 1996; revised manuscript received 7 October 1996)

\begin{abstract}
We study the short-range correlations in a double-quantum-wire structure within the self-consistent scheme of Singwi et al. [Phys. Rev. 179, 589 (1968)]. The local-field factors and static correlation functions are calculated for electron and electron-hole double-wire systems. The ground-state energy and collective excitations are discussed. It is found that the interwire correlations become quite important for electron-hole systems. [S0163-1829(97)03212-8]
\end{abstract}

The advances in fabrication techniques have made it possible to study the quasi-one-dimensional (Q1D) electron systems in semiconducting structures, in which the electrons are confined to move freely only in one dimension. Experimental and theoretical works continue to be of interest, the main motivation coming from their technological potential such as high-speed electronic devices. In this paper we study the ground-state correlations of a double-quantum-wire system at zero temperature. Such structures, analogous to the double-quantum-well systems recently studied, are important in our understanding of the correlation effects in low dimensions. Collective excitations in quantum-wire systems were experimentally studied by spectroscopic methods. ${ }^{1}$ Various aspects of Q1D structures have been investigated in connection with GaAs-based materials. ${ }^{2-6}$ The success of the random-phase approximation (RPA) in interpreting the excitation spectra of quantum wires is attributed ${ }^{3,7}$ to the limited phase space of Q1D systems. The applicability of the Fermiliquid approach (vis á vis the Tomonaga-Luttinger picture ${ }^{8}$ ) to the semiconducting quantum-wire systems has been discussed in detail. ${ }^{9}$ These predictions are in very good agreement with the experimental observation ${ }^{1}$ of collective excitations in GaAs quantum wires. The ground-state correlation effects in single-quantum wires were explored ${ }^{10}$ going beyond the RPA. To include corrections due to exchangecorrelation (xc) effects associated with the charge fluctuations, the method of Singwi et al. ${ }^{11}$ (STLS) offers a physically motivated improvement over the RPA.
Our aim in this paper is to develop the self-consistent scheme of Singwi et al. ${ }^{11}$ to calculate xc effects in doublequantum-wire systems. We specialize in equal density electron and electron-hole (one wire has electrons as charge carriers, whereas the other has holes) systems to study the effects of intra- and interwire correlations. The presence of additional charges in the second quantum wire enhances the correlation effects compared with the case of a single wire. Intra- and interwire correlations are different in nature because the charge carriers can move only in their respective wires (without tunneling) and exchange interactions become important. Interwire correlations increase with decreasing wire separation. The approximation of STLS has proved very useful in double-layer two-dimensional electron-gas systems. ${ }^{12-14}$ The RPA has been found to overestimate the static properties. On the other hand, the method of STLS is believed to give reliable results if the carrier density is not very low. In this work we concentrate on the fully selfconsistent evaluation of the static structure factors and localfield corrections in electron and electron-hole double-wire systems.

We assume that the Q1D electrons in each wire are embedded in a uniform positive background to maintain charge neutrality. The density-density response function of a double-wire system in its extension to a multicomponent case is given by ${ }^{13,15}$

$$
[\chi(q, \omega)]^{-1}=\left(\begin{array}{cc}
{\left[\chi_{11}^{0}(q, \omega)\right]^{-1}-V_{11}(q)\left[1-G_{11}(q)\right]} & -V_{12}(q)\left[1-G_{12}(q)\right] \\
-V_{21}(q)\left[1-G_{21}(q)\right] & {\left[\chi_{22}^{0}(q, \omega)\right]^{-1}-V_{22}(q)\left[1-G_{22}(q)\right]}
\end{array}\right)
$$

where $\chi_{i i}^{0}(q, \omega)$ is the zero-temperature $1 \mathrm{D}$ free-electron density-density response function for the $i$ th wire. Actually, we use the particle-number-conserving expression ${ }^{16}$

$$
\chi^{0}(q, \omega ; \gamma)=\frac{(\omega+i \gamma) \chi^{0}(q, \omega+i \gamma)}{\omega+i \gamma\left[\chi^{0}(q, \omega+i \gamma) / \chi^{0}(q, 0)\right]}
$$

to account for the disorder effects through the phenomenological parameter $\gamma$, in order to justify the use of Fermi- liquid approach in Q1D electron systems. The fluctuationdissipation theorem enables us to express the static structure factors $S_{i j}(q)$ in terms of the response functions. $G_{i j}(q)$ are the static local-field factors arising from the short-range Coulomb correlations and the xc effects for the density-density responses. Setting $G_{i j}=0$ in Eq. (1), one recovers the RPA. The $G_{i j}(q)$ in STLS's approach is obtained by decoupling the two-particle distribution function to write it as a product of two one-particle distribution functions multiplied by the 
pair-correlation function. They are given by ${ }^{10,11}$

$$
G_{i j}(q)=-\frac{1}{n} \int_{-\infty}^{\infty} \frac{d k}{2 \pi} \frac{k V_{i j}(k)}{q V_{i j}(q)}\left[S_{i j}(q-k)-\delta_{i j}\right],
$$

where $n$ is the linear electron density assumed to be the same for both wires. In terms of the Fermi wave vector we have $n=2 k_{F} / \pi$. The electron gas parameter is defined as $r_{s}=\pi / 4 k_{F} a_{B}^{*}$, in which $a_{B}^{*}=\epsilon_{0} / e^{2} m^{*}$ is the effective Bohr radius in the semiconducting wire with background dielectric constant $\epsilon_{0}$ and electron effective mass $m^{*}$.

The model we use for the Q1D electron system is developed by Gold and Ghazali. ${ }^{5}$ It consists of two cylindrical quantum wires of radius $R$, each in an infinite potential well and separated by a distance $d(d>2 R)$. We assume that only the lowest subband in each given quantum wire is occupied. The intra- and interwire Coulomb interactions between the particles are given by Gold and Ghazali ${ }^{5}$ and Gold, ${ }^{17}$ respectively. Notable features of this model are such that the intrawire potential behaves as $\sim|\ln (q R)|$ for long wavelengths and the interwire potential as $\sim|\ln (q d)|$, characteristic of various other proposals. The ground-state energy of the double-wire electron system is expressed as the sum of kinetic-energy and xc-energy parts. The kinetic-energy contribution is simply $T=\pi^{2} / 24 r_{s}^{2} \mathrm{Ry} *$, where the energy unit effective Rydberg is defined as $1 \mathrm{Ry} *=e^{2} / 2 \epsilon_{0} a_{B}^{*}$ We use the standard manipulations to express the xc energy as an integral over the coupling constant ${ }^{10,11}$ in which the selfconsistent values of the static structure factors $S_{i j}(q)$ are used.

We solve the set of equations that describe the structure factors and local-field corrections for density-density response in a double-wire system (for both the electron and electron-hole cases) self-consistently. Material parameters for GaAs are used so that $m^{*}=0.07 m_{e}\left(m_{e}\right.$ is the freeelectron mass) and $\epsilon_{0}=12.9$. In the electron-hole double wires we take the electron and hole effective mass ratio $m_{e}^{*} / m_{h}^{*}=0.134$. The phenomenological disorder parameter $\gamma$ we use in the density-density response function does not influence the converged $S_{i j}(q)$ and $G_{i j}(q)$ significantly for $\gamma \lesssim 0.1 E_{F}$. We mention that the particle-number-conserving expression we use has the same form as in more sophisticated approaches, where it is replaced by the wave-vectorand frequency-dependent memory function $\gamma(q, \omega)$. The phenomenological $\gamma$ may be related to the measured mobilities in quantum wires by the usual relaxation-time expression.

The intrawire static structure factor $S_{11}(q)$ is shown for various $r_{s}$ values in Fig. 1(a). For small $r_{s}, S_{11}(q)$ resembles the noninteracting structure factor given by the Hartree-Fock (HF) approximation. As the density is lowered, correlation effects become more important. The Hubbard approximation (HA) to the local-field factor is obtained from Eq. (3), replacing the static structure factor by the HF expression. This yields $G_{i j}(q) \approx \frac{1}{2}\left[V_{i i}\left(\sqrt{q^{2}+k_{F}^{2}}\right)\right] /\left[V_{i i}(q)\right] \delta_{i j}$. The HA takes into account only the Pauli hole around the electrons. We find that $S_{\mathrm{STLS}}(q)$ is considerably different from $S_{\mathrm{RPA}}(q)$ and $S_{\mathrm{HA}}(q)$. Similar behavior of the static structure factor in Q1D systems has been obtained in various other calculations. ${ }^{10}$ The interwire structure factor $S_{12}(q)$ is about an order of magnitude smaller than $S_{11}(q)$ and negative in
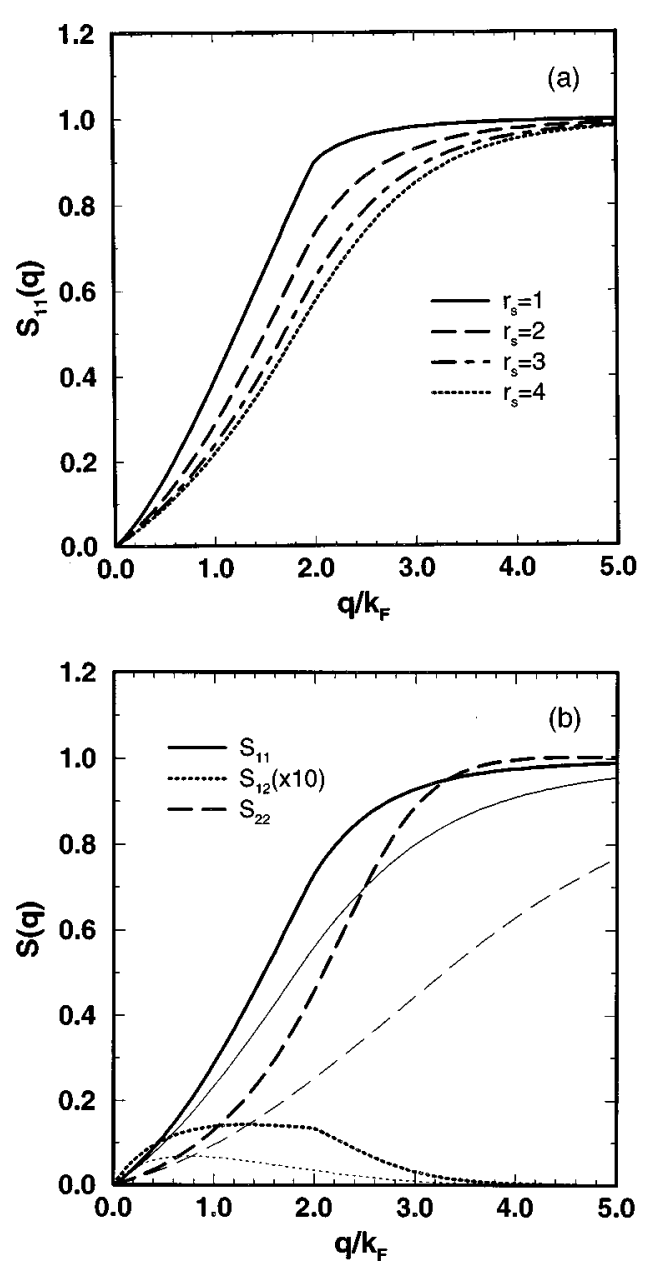

FIG. 1. (a) Intrawire structure factor $S_{11}(q)$ within the method of STLS for a double-wire electron system with $R=2 a_{B}^{*}$ and $d=5 a_{B}^{*}$. The solid, dashed, dot-dashed, and dotted lines are for $r_{s}=1,2,3$, and 4, respectively. (b) $S_{11}(q)$ (solid), $S_{22}(q)$ (dashed), and $S_{12}(q)$ (dotted) in an electron-hole double wire at $r_{s}=2$. The thick and thin lines correspond to the method of STLS and the RPA, respectively.

the range of $q$ values of interest. The RPA for electron-hole systems is even less reliable because the attractive interwire interaction has a larger effect than the repulsive interaction. The failure of the RPA is revealed in the unphysical paircorrelation functions, which are partially remedied in the self-consistent approach. ${ }^{12,13}$ In a multicomponent system, the improvements brought about by STLS's scheme over the RPA are the result of taking multiple scatterings between electrons and holes into account. Although the carrier densities in two wires are kept the same, the differences in the effective masses for electrons and holes render the noninteracting response functions $\chi_{11}^{0}$ and $\chi_{22}^{0}$ different. There is a considerable difference between the self-consistent and RPA calculations. In contrast to the electron double-wire system, the interwire structure factor $S_{12}(q)$ becomes positive. In Fig. 1(b) the intra- and interwire static structure factors $S_{11}(q)$ and $S_{12}(q)$ are shown for different densities.

We summarize the behavior of local-field factor $G_{11}(q)$ in our calculations. As $r_{s}$ increases, the magnitude of $G_{11}(q)$ approaches unity for $q \rightarrow \infty$. In the opposite limit, as $r_{s} \rightarrow 0, G_{11}(q)$ exhibits a peak around $q=2 k_{F}$. We find that 

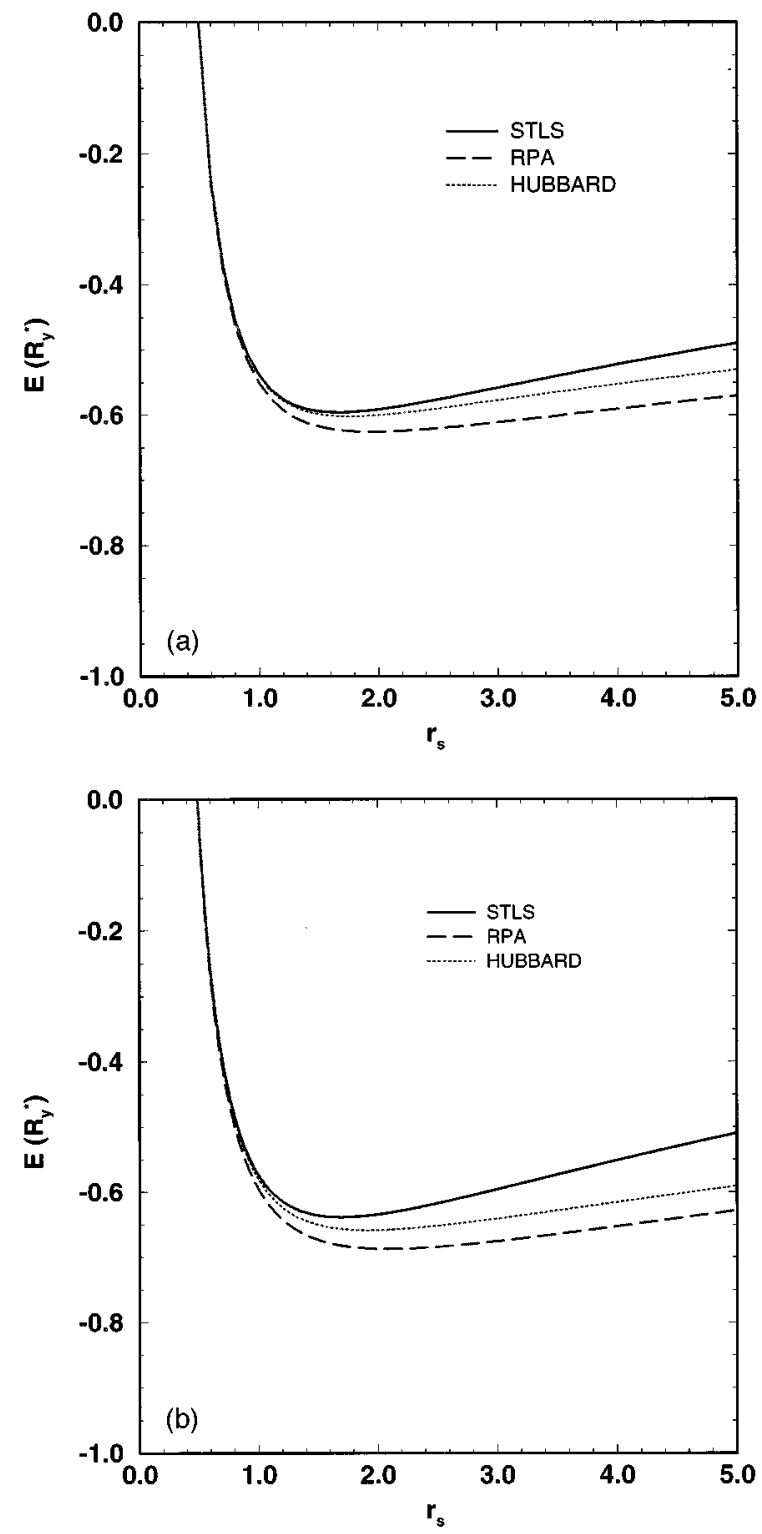

FIG. 2. (a) Ground-state energy of a double-wire electron system as a function of density at a wire separation of $d=5 a_{B}^{*}$. The energies in the method of STLS (solid), the HA (dotted), and the RPA (dashed) are compared for $R=2 a_{B}^{*}$ wires. (b) Same for the double-wire electron-hole system.

$G_{11}$ is not very sensitive to the value of the wire separation $d$ as in the case of double-layer systems. ${ }^{13}$ Our results for $G_{11}(q)$ are in qualitative agreement with the calculations of Wang and Ruden ${ }^{18}$ and single-wire calculations of Friesen and Bergersen. ${ }^{10}$ It should be noted that Wang and Ruden ${ }^{18}$ set $G_{12}(q)=0$, whereas in our calculations both intra- and interwire components of the local-field factor are determined self-consistently. Although the simplification $G_{12}=0$ is justified in electron double-wire systems, in the electron-hole systems it cannot be neglected because of stronger correlations. In an electron-hole double-wire system $G_{12}(q)$ is predominantly negative.

The ground-state energy of a double-wire electron system in different approximations is displayed in Fig. 2(a). We use effective Rydberg as the unit of energy, $1 \mathrm{Ry} *=e^{2} / 2 \epsilon_{0} a_{B}^{*}$. All three curves exhibit minima that lie around $r_{s} \approx 1.5$. The
RPA yields an overestimate for the ground-state energy because the short-range correlation effects are not incorporated. The Hubbard approximation partially remedies this, but $E_{\mathrm{HA}}$ is still below STLS's ground-state energy. Since the interwire interaction decays exponentially (i.e., $\sim e^{-q d}$ ) for large wave vectors, the correlation energy contribution goes to zero as the wire separation $d$ increases. The ground-state energy then becomes the sum of two independent wires. Similar behavior has also been noted for double-layer electron systems. ${ }^{12,13} \mathrm{We}$ find that the ground-state energy does not show a strong dependence on the wire separation for an equal density system with $R=2 a_{B}^{*}$ and $d>5 a_{B}^{*}$. This is mainly because of the weak $d$ dependence of the local-field factor $G_{11}(q)$ discussed above. Nevertheless, for very low densities $\left(r_{s}>5\right)$ it might be possible to have stronger separation distance dependence of the ground-state energies in double-wire systems. More reliable and elaborate approaches would then be required to study this regime. The groundstate energy in an electron-hole double-wire structure is shown in Fig. 2(b). We note that the RPA produces a very loosely bound system (as in the electron double-wire case) since the ground-state energy minimum is less noticeable than those in the other approximations. We observe that the effects of correlations are more important in electron-hole double wires than those in electron systems. The departure from the RPA and HA results become significant for $r_{s}>1$. In general, the ground-state energies are slightly lower (in magnitude) for the electron-hole double wires. We have also calculated the separation dependence of the ground-state energy and found no significant dependence for $d>8 a_{B}^{*}$ in $R=2 a_{B}^{*}$ double-wire systems.

Collective excitations in a double-wire system when correlation effects are included are obtained from the solution of the screening function

$$
\begin{aligned}
\varepsilon(q, \omega)= & {\left[1-V_{11}(q)\left[1-G_{11}(q)\right] \chi_{11}^{0}(q, \omega)\right]\left[1-V_{22}(q)\right.} \\
& \left.\times\left[1-G_{22}(q)\right] \chi_{22}^{0}(q, \omega)\right] \\
& -\left\{V_{12}(q)\left[1-G_{12}(q)\right]\right\}^{2} \chi_{11}^{0}(q, \omega) \chi_{22}^{0}(q, \omega)=0,
\end{aligned}
$$

in which we use the disorder-free response functions. In the case of equal density double-wire electron system the plasmon dispersions are obtained analytically. ${ }^{3}$ The two modes refer to in- and out-of-phase oscillations of the charges and the collective excitations are labeled as the optical and acoustic plasma modes. We show the effects of exchange and correlation described by the local-field factors on the plasmon dispersion of a double-wire electron system in Fig. $3(a)$. The number density in each wire is characterized by $r_{s}=2$, and we take $R=2 a_{B}^{*}$ and $d=5 a_{B}^{*}$. The solid and dotted lines indicate $\omega_{\mathrm{pl}}(q)$ with and without (RPA) the local-field corrections, respectively. The upper and lower (optical and acoustic) plasmon branches merge together at a finite wave vector $q_{c}$ and approach the upper boundary of the particle-hole boundary much faster since the local fields tend to soften the plasmon dispersions. As the separation $d$ between the wires decreases, the interwire correlation effects become more important and $q_{c}$ decreases. For the electronhole double quantum wires, the $q \rightarrow 0$ limit of the dispersion 

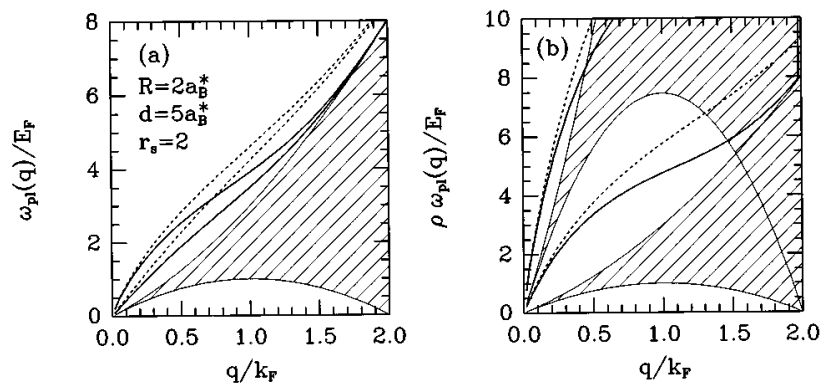

FIG. 3. Plasmon dispersions in (a) a double-wire electron $\left(r_{s}=2\right)$ and (b) an electron-hole $\left(r_{s}=1\right)$ system with $R=2 a_{B}^{*}$ and $d=5 a_{B}^{*}$ The dashed and solid lines stand for the results of the RPA and STLS, respectively.

relations are calculated similarly to the $2 \mathrm{D}$ and Q1D, twocomponent electron-liquid cases. ${ }^{19}$ The difference here is that electron and hole wires are spatially separated. The optical plasmons exist in the region above the single-particle continuum of electrons. We obtain the optical plasmon mode dispersion as $(q \rightarrow 0)$

$$
\left[\omega_{\mathrm{pl}}^{\mathrm{op}}(q)\right]^{2}=B / 2+\left(B^{2} / 4-C\right)^{1 / 2},
$$

where

$$
\begin{gathered}
B=\frac{16 r_{s}}{\pi^{2}} \frac{q^{2}}{\rho}\left[F_{11}\left(1-G_{11}\right) / \rho+F_{11}\left(1-G_{22}\right)\right], \\
C=\frac{16 r_{s}}{\pi^{2}} \frac{q^{4}}{\rho^{3}}\left[F_{11}^{2}\left(1-G_{11}\right)\left(1-G_{22}\right)-F_{12}^{2}\left(1-G_{12}\right)^{2}\right] .
\end{gathered}
$$

In the above expressions, we measure the plasmon energy in terms of the Fermi energy of the holes $\left(E_{F h}=k_{F}^{2} / 2 m_{h}^{*}\right), \rho=m_{e}^{*} / m_{h}^{*}$, and $V_{11}(q)=e^{2} F_{11} / 2 \epsilon_{0}$, etc. Since the mass ratio $1 / \rho \gg 1$, Eq. (4) admits another solution (acoustic plasmon) for energies above the single-particle continuum of holes and below the single-particle continuum of electrons. We find the long-wavelength dispersion of acoustic plasmons to be

$$
\left[\omega_{\mathrm{pl}}^{\mathrm{ac}}(q)\right]^{2}=\frac{\omega_{+}^{2} e^{A^{\prime} / B^{\prime}}-\omega_{-}^{2}}{e^{A^{\prime} / B^{\prime}}-1},
$$

where

$$
\begin{gathered}
A^{\prime}=1-F_{11}\left(1-G_{11}\right)\left(\frac{2 r_{s}}{\pi^{2}}\right) \frac{2}{q} \ln \left|\frac{\omega_{-}}{\omega_{+}}\right|, \\
B^{\prime}=F_{11}\left(1-G_{22}\right)\left(\frac{2 r_{s}}{\pi^{2}}\right) \frac{1}{\rho q}-\left[F_{11}^{2}\left(1-G_{11}\right)\left(1-G_{22}\right)\right. \\
\left.-F_{12}^{2}\left(1-G_{12}\right)^{2}\right]\left(\frac{2 r_{s}}{\pi^{2}}\right)^{2} \frac{2}{\rho q^{2}} \ln \left|\frac{\omega_{-}}{\omega_{+}}\right| .
\end{gathered}
$$

Figure 3(b) shows the optical and acoustic plasmon dispersions calculated using the above long-wavelength expressions in an electron-hole double-wire system at $r_{s}=1$ in which the plasmon energies are scaled with respect to the hole Fermi energy $E_{F h}=\rho E_{F}$. The results of the RPA and STLS are plotted by the dashed and solid lines, respectively. We note that the acoustic plasmon is affected more by the local-field effects than the optical plasmon.

In this work we have considered equal density doublewire systems. Our method can be generalized to include unequal densities. In such cases, it is expected that collective modes will have qualitatively different properties. The semiconducting quantum wires realized so far and used in the experiments are typically in the range $r_{s} \sim 1$. It is conceivable that lower densities can be attained with advances in growth technology. The many-body effects discussed here would then be more readily applicable to the experimental realizations. Charge-density wave-type instabilities discussed in the context of double-quantum-well structures ${ }^{20}$ and also in double-quantum wires ${ }^{17,18}$ could be explored. We have not systematically calculated the static response functions $\chi_{ \pm}(q, 0)$ (obtained by diagonalizing the response matrix $\chi_{i j}$ ) for a wide range of parameters $R, d$, and $r_{s}$, but surmise that interesting features of charge-density wave instability could be studied using our $G_{i j}(q)$.

This work was supported by the Scientific and Technical Research Council of Turkey (TUBITAK) under Grant No. TBAG-AY/77. We thank Professor G. Senatore and Dr. C. Bulutay for useful discussions and acknowledge a helpful communication with Professor D. Neilson.
${ }^{1}$ A. R. Goñi et al., Phys. Rev. Lett. 67, 3298 (1991); A. Schmeller et al., Phys. Rev. B 49, 14778 (1994).

${ }^{2}$ G. Y. Hu and R. F. O'Connell, Phys. Rev. B 42, 1290 (1990).

${ }^{3}$ Q. P. Li and S. Das Sarma, Phys. Rev. B 43, 11768 (1991).

${ }^{4}$ B. Y.-K. Hu and S. Das Sarma, Phys. Rev. Lett. 68, 1750 (1992); Phys. Rev. B 48, 5469 (1993).

${ }^{5}$ A. Gold and A. Ghazali, Phys. Rev. B 41, 7626 (1990).

${ }^{6}$ Y. Sun and G. Kirczenow, Can. J. Phys. 73, 357 (1995).

${ }^{7}$ J.-X. Yu and J.-B. Xia, Solid State Commun. 98, 227 (1996).

${ }^{8}$ J. Sólyom, Adv. Phys. 28, 201 (1979). For a fairly recent review, see, e.g., The Many-Body Problem, edited by D. C. Mattis (World Scientific, Singapore, 1993).

${ }^{9}$ See Ref. 4 for a detailed account of a comparison of Fermi-liquid and Luttinger liquids.

${ }^{10}$ W. I. Friesen and B. Bergersen, J. Phys. C 13, 6627 (1980); V. B. Campos, M. H. Degani, and O. Hipólito, Superlatt. Microstruct. 17, 85 (1995); L. Calmels and A. Gold, Phys. Rev. B 51, 8426 (1995).
${ }^{11}$ K. S. Singwi et al., Phys. Rev. 176, 589 (1968); K. S. Singwi and M. P. Tosi, Solid State Phys., 36, 177 (1981).

${ }^{12}$ L. Zheng and A. H. MacDonald, Phys. Rev. B 49, 5522 (1994).

${ }^{13}$ L. Liu et al., Phys. Rev. B 53, 7923 (1996); J. Szymánski, L. Świerkowski, and D. Neilson, ibid. 50, 11002 (1994).

${ }^{14}$ C. Zhang and N. Tzoar, Phys. Rev. B 38, 5786 (1988); M. Alatalo et al., ibid. 49, 8277 (1994).

${ }^{15}$ A. Sjölander and J. Stott, Phys. Rev. B 5, 2109 (1972).

${ }^{16}$ N. D. Mermin, Phys. Rev. B 1, 2362 (1970); A. K. Das, J. Phys. F 5, 2035 (1975).

${ }^{17}$ A. Gold, Philos. Mag. Lett. 66, 163 (1992).

${ }^{18}$ R. Wang and P. P. Ruden, Phys. Rev. B 52, 7826 (1995).

${ }^{19}$ G. Vignale, Phys. Rev. B 38, 811 (1988); B. Tanatar, Solid State Commun. 92, 699 (1994).

${ }^{20}$ L. Świerkowski, D. Neilson, and J. Szymański, Phys. Rev. Lett. 67, 240 (1991); D. Neilson et al., ibid. 71, 4035 (1993). 